\title{
A inserção de cateter epicutâneo por profissionais de enfermagem na unidade de terapia intensiva neonatal
}

The insertion of epicutaneous catheters by nursing professionals in the neonatal intensive care unit La inserción de catéteres epicutáneos por parte de los profesionales de enfermería em la unidad de cuidados intensivos neonatales

Recebido: 10/06/2021 | Revisado: 18/06/2021 | Aceito: 22/06/2021 | Publicado: 07/07/2021

Lucas Sousa Penha

ORCID: https://orcid.org/0000-0002-6634-1081 Faculdade Santa Terezinha, Brasil

E-mail: lucassousasales2014@gmail.com

Vitoria Vilas Boas da Silva Bomfim

ORCID: https://orcid.org/0000-0003-4897-0279

Centro Universitário Jorge Amado, Brasil E-mail: pesquisaclinica9@gmail.com

Laiane da Silva Lima Oliveira

ORCID: https://orcid.org/0000-0001-5531-7636 Centro Universitário Jorge Amado, Brasil E-mail: laianeolima11@gmail.com

Emerson Galdino Rodrigues dos Santos ORCID: https://orcid.org/0000-0003-2290-4759

Faculdade de Tecnologia e Ciências, Brasil E-mail: galdinoegrs@gmail.com Larissa Araújo Torres ORCID: https://orcid.org/0000-0003-3235-5802 Universidade de Brasília, Brasil E-mail: larissaa.torres@hotmail.com

Maria Dhescyca Ingrid Silva Arruda

ORCID: https://orcid.org/0000-0002-9073-7844

Faculdade São Francisco da Paraíba, Brasil E-mail: dhescycaingrid20@gmail.com Sandy Sá Menezes Abreu ORCID: https://orcid.org/0000-0002-5736-6551 Faculdade Santa Terezinha, Brasil E-mail: samenezessandy@gmail.com

Maria Rosemary da Silva Gomes ORCID: https://orcid.org/0000-0002-8217-3647

Centro Universitário Santo Agostinho, Brasil

E-mail: mariarosemary2010@gmail.com

Sirlene Aparecida de Oliveira

ORCID: https://orcid.org/0000-0003-2352-7983

Faculdade de Ensino Superior de Cacoal, Brasil

E-mail: sirleneaparecidadeoliveira1988@gmail.com

Karennfher Cimas Alves

ORCID: https://orcid.org/0000-0001-8266-2021

Faculdade Estácio de Sá, Brasil

E-mail: karencimas@hotmail.com

Aglaya Oliveira Lima Cordeiro de Almeida

ORCID: https://orcid.org/0000-0002-8268-3380 Universidade Federal da Bahia, Brasil E-mail: aglayatriz@gmail.com

Graziele Melo da Silva

ORCID: https://orcid.org/0000-0002-5896-1344

Universidade Castelo Branco, Brasil

E-mail: grazymelo428@gmail.com

Jackson Gois Teixeira

ORCID: https://orcid.org/0000-0003-2804-1130

Centro Universitário do Destrito Federal, Brasil

E-mail: jacksonudf1@gmail.com

Vitória Campos dos Santos

ORCID: https://orcid.org/0000-0002-1271-9253

Universidade Salvador, Brasil

E-mail: enfvitoriacampos19999@gmail.com 


\begin{abstract}
Resumo
Objetivos: Avaliar a inserção de cateter epicutâneo por profissionais de enfermagem e seus riscos e complicações. Métodos: Revisão integrativa de literatura, realizada nas bibliotecas de dados BVS e PubMed, utilizando as bases de dados LILACS, MEDLINE e BDENF, com o intuito de buscar artigos disponíveis em português, inglês e espanhol, que foram publicados entre o período de janeiro de 2015 e junho de 2021. Resultados: Foram encontrados na busca de literatura 160 artigos, sendo incluídos 9 artigos na amostra final. A utilização do Cateter de Central de Inserção Periférica (PICC) é muito utilizado em recém-nascidos prematuros, devido à sua imaturidade e dificuldade em manter a boa funcionalidade dos órgãos vitais. Durante a inserção do PICC, alguns profissionais administram sedativos leves ou analgésicos, porém um estudo mostrou que o tratamento não farmacológico para alívio da dor também é muito eficaz e é ofertado soluções adocicadas ou Leite Materno. Considerações Finais: Diante do exposto, podemos notar que o cateter de PICC é a melhor opção para recém nascidos internados na UTI Neonatal que necessitam de terapia de infusão contínua, antibioticoterapia e nutrição parenteral.
\end{abstract}

Palavras-chave: Cateterismo venoso central; Unidade de terapia intensiva neonatal; Cuidados de enfermagem.

\begin{abstract}
Objectives: To evaluate the insertion of epicutaneous catheters by nursing professionals and its risks and complications. Methods: Integrative literature review, carried out in the BVS and PubMed data libraries, using the LILACS, MEDLINE and BDENF databases, in order to search for articles available in Portuguese, English and Spanish, which were published between the period of January of 2015 and June 2021. Results: 160 articles were found in the literature search, including 9 articles in the final sample. The use of the Peripherally Inserted Central Catheter (PICC) is widely used in premature newborns, due to its immaturity and difficulty in maintaining the good functionality of vital organs. During the insertion of the PICC, some professionals administer mild sedatives or analgesics, but a study has shown that non-pharmacological treatment for pain relief is also very effective and sweetened solutions or breast milk are offered. Final Considerations: Given the above, we can note that the PICC catheter is the best option for newborns admitted to the Neonatal ICU who need continuous infusion therapy, antibiotic therapy and parenteral nutrition.
\end{abstract}

Keywords: Central venous catheterization; Neonatal intensive care unit; Nursing care.

\title{
Resumen
}

Objetivos: Evaluar la inserción de catéteres epicutáneos por profesionales de enfermería y sus riesgos y complicaciones. Métodos: Revisión integrativa de la literatura, realizada en las bibliotecas de datos BVS y PubMed, utilizando las bases de datos LILACS, MEDLINE y BDENF, con el fin de buscar artículos disponibles en portugués, inglés y español, que fueron publicados entre el período de enero de 2015 y Junio de 2021. Resultados: Se encontraron 160 artículos en la búsqueda de literatura, incluyendo 9 artículos en la muestra final. El uso de Catéter Central de Inserción Periférica (CCIP) es muy utilizado en recién nacidos prematuros, debido a su inmadurez y dificultad para mantener la buena funcionalidad de órganos vitales. Durante la inserción del PICC, algunos profesionales administran sedantes o analgésicos suaves, pero un estudio ha demostrado que el tratamiento no farmacológico para el alivio del dolor también es muy efectivo y se ofrecen soluciones endulzadas o leche materna. Considerciones Finales: Dado lo anterior, podemos señalar que el catéter PICC es la mejor opción para los recién nacidos ingresados en la UCI Neonatal que necesitan terapia de infusión continua, terapia antibiótica y nutrición parenteral.

Palabras clave: Cateterismo venoso central; Unidad de cuidados intensivos neonatales; Cuidados de enfermería.

\section{Introdução}

No Brasil, muitos nascimentos ocorrem de forma prematura. E devido a prematuridade, muitos desses recém-nascidos (RN) dependem de suporte intensivo para que realizem suas funções vitais, sendo encaminhados para a Unidade de Terapia Intensiva Neonatal (UTIN). Dotada de alta tecnologia e cuidados intensivos, esta unidade visa manter as funções vitais do RN estáveis, visto que os mesmos não possuem maturação nos mecanismos de autorregulação clínica (Marski, Facio, Ichisato, Barba \& Wernet, 2018; Ferreira, Magalhães, Rolim, da Silva, da Silva, Sampaio \& de Almeida Freitas, 2018).

Como a maioria das soluções e medicações no paciente recém-nascido é feita por via endovenosa, um dos procedimentos mais realizados nos RN’s na UTIN são as punções venosas. Porém, tais pacientes possuem particularidades anatômicas como uma rede venosa frágil e características cutâneas diferenciadas, tendo como consequência uma curta permanência dos acessos venosos. Além disso, potenciais complicações como infiltrações e flebites podem tornar esse acesso inviável, dificultando o processo de reabilitação do neonato (Jantsch, Neves, Arrué, Kegler \& de Oliveira, 2014; Leiman, Issi, Ludwig, Ferreira \& Algeri, 2019). 
Baseado nessas peculiaridades anatômicas e as possíveis complicações dos cateteres venosos periféricos em recémnascidos, têm-se como alternativa a utilização do Cateter Central de Inserção Periférica ou Cateter Epicutâneo (Jantsch et al., 2014; Silva, 2018). Esse cateter cada vez mais utilizado nas unidades neonatais e pediátricas possui inúmeras vantagens, dentre elas: facilidade na inserção, prolongado tempo de permanência, menos traumas na inserção e menor risco de complicações (dos Reis Corrêa, Manzo, Matozinhos, Carvalho, dos Santos \& Gomes, 2019)

O Cateter Central de Inserção Periférica, ou PICC como é mais conhecido, trata-se de um dispositivo vascular formado por compostos biocompatíveis inserido com ajuda de uma agulha introdutora em uma veia superficial, sendo as mais utilizadas as veias cefálicas e basílica, até a veia cava superior tornando-se ali um acesso central. Sua inserção pode acontecer a beira leito com uso de anestesia local, não necessitando de procedimento cirúrgico (Montes, Teixeira, Barbosa \& Barichello, 2011).

Porém, apesar de ser um dispositivo seguro e eficaz no tratamento de recém-nascidos internados em UTIN, o Cateter Epicutâneo não está livre de complicações, sendo as principais relacionadas ao seu uso: a flebite, o mau posicionamento, trombose, oclusãoe sepse. Além do mais, complicações como consequência da inaptidão na inserção de tal cateter também é possível, devendo o mesmo ser inserido por profissionais habilitados e com prática para tal função (dos Reis Corrêa et al., 2019; Montes, Teixeira, Barbosa \& Barichello, 2011; Chopra, Ratz, Kuhn, Lopus, Chenoweth \& Krein, 2014).

Segundo o Conselho Federal de Enfermagem (COFEN) através da Resolução 258/2011, é lícito a inserção do Cateter Venoso Central de Inserção Periférica pelo profissional de Enfermagem, desde que o mesmo possua qualificação e/ou capacitação profissional para isso (Assis, Mota, Cesar, Turrini \& Ferreira, 2021; das Neves Motta, Fialho, Dias \& do Nascimento, 2011). Desta maneira, considerando-se os riscos, complicações e técnicas que devem ser utilizadas para realizar o procedimento, o presente estudo objetiva avaliar a inserção de cateter epicutâneo por profissionais de enfermagem e seus riscos e complicações.

\section{Metodologia}

Trata-se de uma revisão integrativa de literatura de abordagem quantitativa (Ludke, 2013). Realizada nas bibliotecas de dados Biblioteca Virtual de Saúde (BVS) e U.S. National Library of Medicine (PubMed), utilizando as bases de dados Literatura Latino-Americana e do Caribe em Ciências da Saúde (LILACS), Medical Literature Analysis and Retrieval System Online (MEDLINE), Base de Dados de Enfermagem (BDENF), com o intuito de buscar artigos disponíveis na íntegra em português, inglês e espanhol, que foram publicados entre o período de janeiro de 2015 e junho de 2021, sendo justificado a escolha do período a fim de incluir uma amostra mais atual. Utilizados os descritores em Ciências de Saúde (DeCS): "Cateterismo Venoso Central", "Unidade de Terapia Intensiva Neonatal" e "Cuidados de Enfermagem", por meio da equação booleana ("Cateterismo Venoso Central" AND "Unidade de Terapia Intensiva Neonatal" AND "Cuidados de Enfermagem") para busca na BVS e com uso dos Medical Subject Headings (MeSH): "Catheterization, Central Venous", "Intensive Care Units, Neonatal" e "Nursing Care", por meio da equação booleana ("Catheterization, Central Venous" AND "Intensive Care Units, Neonatal" AND ”Nursing Care”) para busca na PubMed. Foram excluídos artigos repetidos nas bases de dados. 
Figura 1 - Fluxograma de busca dos artigos. Brasil, 2021.

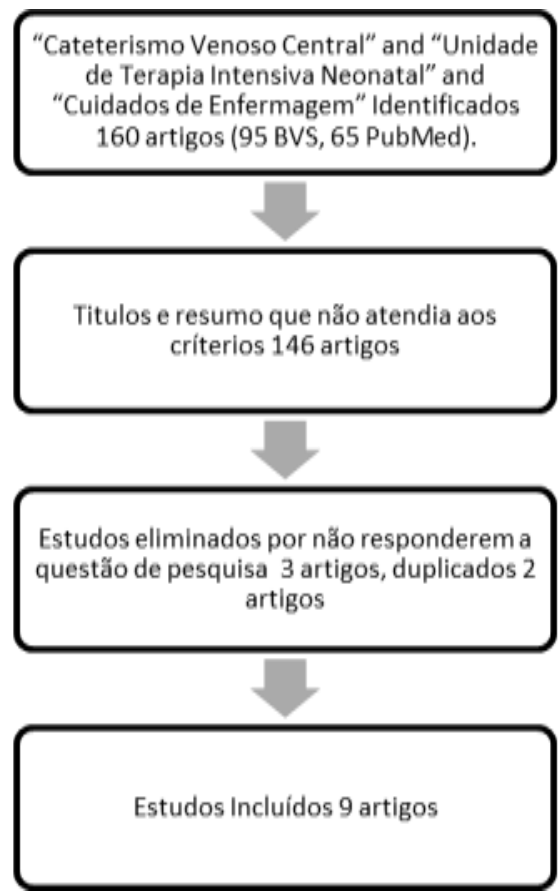

Fonte: Autores (2021).

\section{Resultados e Discussão}

Foram encontrados na busca de literatura 160 artigos (95 BVS e 65 PubMed), nos quais após a aplicação dos critérios de inclusão e exclusão restaram 36 artigos (30 BVS e 6 PubMed) para a revisão em pares. Após a revisão em pares ficaram 16 artigos a serem lidos na íntegra, sendo assim foram incluídos 9 artigos conforme Quadro 1.

Quadro 1 - Artigos incluídos na amostra final. Brasil, 2021.

\begin{tabular}{|c|c|c|c|c|}
\hline Número & Autor e Ano & Periódico & Objetivo & Resultado \\
\hline 1 & $\begin{array}{l}\text { Pereira, Makuch, } \\
\text { Freitas, Secco \& } \\
\text { Danski (2020) }\end{array}$ & $\begin{array}{l}\text { Enfermagem em } \\
\text { foco }\end{array}$ & $\begin{array}{c}\text { Identificar o conhecimento dos } \\
\text { enfermeiros quanto à inserção e } \\
\text { manutenção do PICC em uma UTIN } \\
\text { de um hospital pediátrico de } \\
\text { referência. }\end{array}$ & $\begin{array}{l}\text { A maioria dos profissionais entrevistados } \\
\text { possuem habilitação e apresentaram boas } \\
\text { práticas para inserção de PICC; realizam } \\
\text { manutenção de cateter (curativos, trocas). }\end{array}$ \\
\hline 2 & $\begin{array}{l}\text { Pereira Ferreira, C., } \\
\text { Lemos Querido, D., } \\
\text { Moreira Christoffel, } \\
\text { M., Saraiva de } \\
\text { Almeida, Andrade \& } \\
\text { Camilo Leite (2020) }\end{array}$ & $\begin{array}{l}\text { Revista } \\
\text { Eletrônica de } \\
\text { Enfermagem }\end{array}$ & $\begin{array}{c}\text { Analisar a utilização do PICC em } \\
\text { recém-nascidos internados em uma } \\
\text { Unidade de Terapia } \\
\text { Intensiva Neonatal. }\end{array}$ & $\begin{array}{l}\text { O sexo foi predominante masculino, maior } \\
\text { causa de uso de PICC foi a prematuridade, } \\
\text { novas inserções foram necessárias devido } \\
\text { eventos adversos como hiperemia e } \\
\text { cordão palpável, tempo de permanência } \\
\text { em média foi entre } 5 \text { a } 10 \text { dias. }\end{array}$ \\
\hline 3 & $\begin{array}{l}\text { Mittang, Stiegler, } \\
\text { Schultz \& Kroll } \\
\text { (2020) }\end{array}$ & $\begin{array}{l}\text { Revista Baiana } \\
\text { de Enfermagem }\end{array}$ & $\begin{array}{l}\text { Identificar os principais fatores de } \\
\text { retirada do cateter central de inserção } \\
\text { periférica em recém-nascidos em uma } \\
\text { unidade de terapia intensiva neonatal e } \\
\text { verificar a associação de variáveis } \\
\text { relacionadas ao recém-nascido e ao } \\
\text { cateter central com os fatores de } \\
\text { retirada. }\end{array}$ & $\begin{array}{l}\text { Entre as complicações associadas à } \\
\text { retirada do PICC estão infecção do cateter } \\
\text { e flebite, rompimento, expulsão acidental, } \\
\text { dificuldade em observar a ponta do } \\
\text { cateter, obstrução e infiltração. }\end{array}$ \\
\hline
\end{tabular}




\begin{tabular}{|c|c|c|c|c|}
\hline 4 & $\begin{array}{l}\text { Baggio, Cheffer, da } \\
\text { Luz, de Marchi } \\
\text { Sanches \& Berres } \\
\text { (2019) }\end{array}$ & Revista Rene & $\begin{array}{l}\text { Analisar a utilização do cateter central } \\
\text { de inserção periférica em neonatos. }\end{array}$ & $\begin{array}{l}\text { A maioria das indicações para uso de } \\
\text { PICC foi para complicações da } \\
\text { prematuridade como uso de } \\
\text { antibioticoterapia e nutrição parenteral. }\end{array}$ \\
\hline 5 & $\begin{array}{c}\text { Rangel, Castro, } \\
\text { Amorim, } \\
\text { Zandonade, } \\
\text { Christoffel \& Primo } \\
\text { (2019) }\end{array}$ & $\begin{array}{l}\text { Rev. Fund. Care } \\
\text { Online }\end{array}$ & $\begin{array}{l}\text { Avaliar as práticas de enfermagem na } \\
\text { inserção, manutenção } \\
\text { e remoção do PICC em neonatos. }\end{array}$ & $\begin{array}{l}\text { A maioria dos RN's eram do sexo } \\
\text { feminino, prematuros, em uso de } \\
\text { hidratação venosa, antibioticoterapia, } \\
\text { nutrição enteral, a maioria sofreu } \\
\text { múltiplas punções, as veias mais } \\
\text { puncionadas foram basílica e cefálica. } \\
\text { Houve uso de analgésico durante inserção } \\
\text { de PICC, tendo a posição do cateter } \\
\text { adequada e confirmada através do exame } \\
\text { radiográfico. Houve, em média, troca de } \\
\text { curativo } 3 \text { vezes por paciente, e a cultura } \\
\text { de } 9 \text { cateteres detectou } 5 \text { com resultados } \\
\text { positivos para contaminação. }\end{array}$ \\
\hline 6 & $\begin{array}{l}\text { Borghesan, Demitto, } \\
\text { Fonseca, Fernandes, } \\
\text { Costenaro \& } \\
\text { Higarashi (2017) }\end{array}$ & $\begin{array}{l}\text { Revista de } \\
\text { Enfermagem } \\
\text { UFRJ }\end{array}$ & $\begin{array}{l}\text { Traçar o perfil de indicação e } \\
\text { utilização do PICC na realidade } \\
\text { assistencial da unidade de terapia } \\
\text { intensiva (UTIN) de um hospital } \\
\text { universitário do sul do Brasil. }\end{array}$ & $\begin{array}{l}\text { A maioria dos RN's são prematuros, do } \\
\text { sexo masculino, com asfixia de leve a } \\
\text { grave segundo índice de Apgar no } \\
\text { primeiro minuto de vida. O uso de PICC } \\
\text { foi mais recomendado para nutrição } \\
\text { parenteral, havendo mais de uma inserção } \\
\text { por tempo de internação aumentado. } \\
\text { Menos da metade utilizaram medidas de } \\
\text { analgesia durante inserção de PICC, } \\
\text { porém houve administração de soluções } \\
\text { adocicadas e Leite Materno. Utilizou-se } \\
\text { contenção facilitada durante o } \\
\text { procedimento, e as veias preferíveis foram } \\
\text { cubital e safena, e como última escolha a } \\
\text { veia axilar. Verificou-se que a maneira } \\
\text { correta de inserir a ponta do PICC é de } \\
\text { fundamental importância no } \\
\text { procedimento. }\end{array}$ \\
\hline 7 & $\begin{array}{l}\text { Nobre, Cardoso, } \\
\text { Teixeira, Lopes \& } \\
\text { Fontenele (2016) }\end{array}$ & $\begin{array}{l}\text { Online Brazilian } \\
\text { Journal of } \\
\text { Nursing }\end{array}$ & $\begin{array}{l}\text { Analisar a utilização do cateter central } \\
\text { de inserção periférica quanto aos } \\
\text { aspectos da técnica de inserção, } \\
\text { posicionamento e manutenção do } \\
\text { cateter, assim como a influência no } \\
\text { número de dissecções venosas em } \\
\text { bebês internados em unidade neonatal. }\end{array}$ & $\begin{array}{l}\text { Predominaram bebês com desconforto } \\
\text { respiratório precoce e prematuridade, do } \\
\text { sexo masculino, indicados para } \\
\text { antibioticoterapia e nutrição parenteral. } \\
\text { Predominância das veias basílica e } \\
\text { cefálica e com posicionamento inicial da } \\
\text { ponta central. }\end{array}$ \\
\hline 8 & $\begin{array}{c}\text { Souza, Alves, } \\
\text { Rodrigues, Dames, } \\
\text { Medeiros \& Paiva } \\
\text { (2016) }\end{array}$ & $\begin{array}{l}\text { Online Brazilian } \\
\text { Journal of } \\
\text { Nursing }\end{array}$ & $\begin{array}{c}\text { Analisar o conhecimento dos } \\
\text { enfermeiros da UTIN acerca da } \\
\text { inserção, manuseio, manutenção e } \\
\text { retirada do cateter central de inserção } \\
\text { periférica. }\end{array}$ & $\begin{array}{c}\text { Os profissionais entrevistados } \\
\text { demonstraram conhecimento teórico- } \\
\text { prático para realizar o procedimento de } \\
\text { inserção de PICC. Explanaram a } \\
\text { importância da higienização das mãos e } \\
\text { antissepsia para evitar infecçães, maneira } \\
\text { de inserir a ponta do cateter, avaliação da } \\
\text { necessidade e do local de punção, a } \\
\text { importância de implementar um POP, } \\
\text { troca de curativos e informaram que } \\
\text { mesmo invasivo, o PICC tem muitas } \\
\text { vantagens que devem ser consideradas } \\
\text { (tempo de duração, menor manipulação do } \\
\text { bebê, redução de estresse por diminuir as } \\
\text { punções, proteção e menor taxa de } \\
\text { contaminação se realizado corretamente. }\end{array}$ \\
\hline
\end{tabular}




\begin{tabular}{|c|c|c|c|c|}
\hline & De Luca (2015) & $\begin{array}{c}\text { Faculdade de } \\
\text { Enfermagem } \\
\text { UERJ }\end{array}$ & $\begin{array}{c}\text { Conhecer o significado de segurança } \\
\text { para o enfermeiro no processo de } \\
\text { utilização do PICC em recém- } \\
\text { nascidos; descrever os cuidados } \\
\text { prestados pelo enfermeiro no uso do } \\
\text { PICC em recém-nascidos e analisar os } \\
\text { nexos entre segurança e os princípios } \\
\text { configura pelo tempo de duração da } \\
\text { nascidos no uso do PICC em recém- } \\
\text { terapia prolongada (mais de uma semana), } \\
\text { ou devido à nutrição parenteral, } \\
\text { antibioticoterapia ou quimioterápicos. O } \\
\text { PICC reduz o desconforto causado pelas } \\
\text { várias punções. Os entrevistados } \\
\text { informam a importância do diálogo entre a } \\
\text { equipe de enfermagem e médica, avaliar o } \\
\text { quadro clínico do RN antes da tomada de } \\
\text { decisão, os cuidados pré, durante e após o } \\
\text { procedimento são fundamentais.E dizem } \\
\text { que o planejamento é essencial. }\end{array}$ \\
\hline
\end{tabular}

Fonte: Autores (2021).

Os artigos 4, 5, 7 e 9 abordam sobre a utilização do Cateter de Central de Inserção Periférica (PICC) que é muito utilizado em recém-nascidos prematuros, devido à sua imaturidade e dificuldade em manter a boa funcionalidade dos órgãos vitais. Desta maneira, este público necessita de um suporte que o auxilie no seu desenvolvimento. Sua indicação é para RN's que venham a utilizar infusões por um longo período, ou seja, acima de uma semana; que estejam, principalmente, em uso de antibioticoterapia e nutrição parenteral (Baggio et al., 2019; Rangel et al., 2019; Nobre et al., 2016; De Luca, 2015).

Nos artigos 8 e 9 os autores trazem que o PICC reduz a dor e o desconforto causados pelo excesso de punções venosas, e mesmo sendo um procedimento invasivo, possui inúmeras vantagens tais como: diminuição da quantidade de punções venosas, ou seja, diminui a manipulação do RN, diminui as taxas de estresse, promove proteção e reduz as chances de contaminação se manuseado na maneira correta (Souza et al., 2016; De Luca, 2015).

Durante a inserção do PICC, alguns profissionais administram sedativos leves ou analgésicos, como se vê nos artigos 5 e 6, porém o estudo 6 ainda mostrou que o tratamento não farmacológico para alívio da dor também é muito eficaz e é ofertado soluções adocicadas ou Leite Materno. O que reflete na humanização do cuidado e promoção do bem-estar tanto do paciente quanto da família (Rangel et al., 2019; Borghesan et al., 2017).

No primeiro e oitavo artigos notou-se que a maioria dos profissionais se mostraram conhecedores da teoria e da prática quando se trata de inserção de PICC, podendo-se perceber que com a implementação do POP (Procedimento Operacional Padrão) a segurança e confiabilidade dos profissionais se fez garantida, transpassando para os familiares do paciente pois, sabe-se que a falta desse protocolo induz à desorganização e ao erro (Pereira et al., 2020; Souza et al., 2016).

No artigo 1, onde foi feito um levantamento descritivo quanto ao nível de conhecimento dos profissionais de enfermagem quanto a inserção e manutenção do PICC, foi observado que a maioria dos profissionais que inserem esse cateter possui curso de habilitação para tal, conforme é recomendado pelos órgãos da classe. Além disso, o estudo evidenciou que a troca de curativos a cada sete dias ou que apresentem sujidade, umidade, sangramento ou bordas com pouca adesão. A utilização de películas transparentes semipermeáveis é recomendada, pois a mesma possui a vantagem de permitir a visualização do sítio de inserção do cateter, atua como barreira contra microrganismos, aumenta o tempo de permanência do cateter e proporciona maior conforto ao paciente, além de ser de menor custo para a instituição (Pereira et al., 2020).

Puderam ser observadas no estudo 9 que a avaliação do quadro clínico do RN, o planejamento do procedimento e a comunicação efetiva entre as equipes são passos fundamentais para prestar uma assistência de qualidade e eficaz, dessa forma, os profissionais de enfermagem observaram a situação paciente, avaliaram os riscos e benefícios propostos pelos princípios bioéticos, observaram o local para inserção do cateter, realizaram o planejamento e dialogaram com seus colegas, para enfim realizar o procedimento seguindo as técnicas assépticas como medida de prevenção de sítio de infecção nosocomial (De Luca, 2015). 
Depois de inserido o cateter, as avaliações são baseadas no curativo, local de inserção com presença de edema, hiperemia, extravazamento ou infiltração, flebite, rompimento, expulsão acidental, como pôde ser visualizado nos artigos 2 e 3 . Caso haja alguma dessas complicações, é necessário realizar outra avaliação para inserir o PICC novamente. Vale salientar a importância de solicitar um exame radiográfico (Raio-X) para avaliar o posicionamento correto do PICC a fim de evitar tais complicações, contudo, apenas o estudo 5 abordou esta técnica (Saraiva de Almeida et al., 2020; Mittang et al., 2020; Rangel et al., 2019).

\section{Considerações Finais}

Diante do exposto, podemos notar que o cateter de PICC é a melhor opção para recém nascidos internados na UTI Neonatal que necessitam de terapia de infusão contínua, antibioticoterapia e nutrição parenteral. Porém cuidados devem ser tomados como a técnica estéril durante todo o procedimento, verificar validade do cateter, raio-x de controle. Quanto a sua inserção é uma prática atribuída ao enfermeiro que deve possuir habilitação técnica para tal. Sendo esse profissional também responsável pela manutenção do mesmo, por educar sua equipe quanto ao manuseio do cateter. Caso tal procedimento seja realizado com eficácia e segurança, e a assistência prestada seja de qualidade, os índices de bom prognóstico de neonatos internados na Unidade de Terapia Intensiva Neonatal será muito maior.

\section{Referências}

Assis, G. L. C. D., Mota, A. N. B., Cesar, V. F., Turrini, R. N. T., \& Ferreira, L. M. (2021). Custo direto da inserção do Cateter Central de Inserção Periférica por enfermeiros em adultos hospitalizados. Revista Brasileira de Enfermagem, 74.

Baggio, M. A., Cheffer, M. H., da Luz, M. A. P., de Marchi Sanches, M., \& Berres, R. (2019). Use of the central catheter for peripheral insertion in newborns: analysis of indication for removal. Rev Rene, (20), 48.

Borghesan, N. B. A., Demitto, M. O., Fonseca, L. M. M., Fernandes, C. A. M., Costenaro, R. G. S., \& Higarashi, I. H. (2017). Cateter venoso central de inserção periférica: práticas da equipe de enfermagem na atenção intensiva neonatal [Peripherally inserted central catheter: practics of nursing team in the neonatal intensive care][Catéter central de inserción periférica: práctica del equipo de enfermería en la atención intensiva neonatal]. Revista Enfermagem UERJ, 25, 28143.

Chopra, V., Ratz, D., Kuhn, L., Lopus, T., Chenoweth, C., \& Krein, S. (2014). PICC-associated bloodstream infections: prevalence, patterns, and predictors. The American journal of medicine, 127(4), 319-328.

das Neves Motta, P., Fialho, F. A., Dias, I. M. Á. V., \& do Nascimento, L. (2011). Cateter central de inserção periférica: o papel da enfermagem na sua utilização em neonatologia. HU Revista, 37(2).

De Luca, H. M. (2015). O enfermeiro e a utilização do cateter central de inserção periférica em recém-nascidos: contribuições à luz da segurança e bioética.

dos Reis Corrêa, A., Manzo, B. F., Matozinhos, F. P., Carvalho, F. C. F., dos Santos, L. B., \& Gomes, M. L. (2019). Influência do tempo de atuação do enfermeiro no conhecimento sobre cateter epicutâneo: estudo transversal. Online Brazilian Journal of Nursing, 18(4).

Ferreira, L. A., Magalhães, F. J., Rolim, K. M. C., da Silva, F. E. J. A., da Silva, W. P. G., Sampaio, R. G., \& de Almeida Freitas, G. K. L. (2018). Intervenções de Enfermagem no uso do PICC em pediatria e neonatologia: evidências científicas. CIAIQ2018, 2.

Jantsch, L. B., Neves, E. T., Arrué, A. M., Kegler, J. J., \& de Oliveira, C. R. (2014). Utilização do cateter central de inserção periférica em neonatologia. Revista Baiana de Enfermagem28,(3).

Leiman, S. H., Issi, H. B., Ludwig, M. C. F., Ferreira, A. M., \& Algeri, S. (2019). Implicações para prática de enfermagem no uso do cateter central de inserção periférica (PICC) em oncologia pediátrica no ambiente extra-hospitalar. Anais. Semana de Enfermagem.

Ludke, M. A. (2013). MEDA de. Pesquisa em educação: abordagens qualitativas. EPU.

Marski, B. D. S. L., Facio, B. C., Ichisato, S. M. T., Barba, P. C. D. S. D., \& Wernet, M. (2018). Cuidado Desenvolvimental: assistência de enfermeiros de Unidade de Terapia Intensiva Neonatal. Revista Brasileira de Enfermagem, 71, 2758-2766.

Mittang, B. T., Stiegler, G., Schultz, L. F., \& Kroll, C. (2020). Fatores Para Retirada De Cateter Central De Inserção Periférica Em Unidade De Terapia Intensiva Neonatal. Revista Baiana de Enfermagem34,.

Montes, S. F., Teixeira, J. B. A., Barbosa, M. H., \& Barichello, E. (2011). Ocorrência de complicações relacionadas ao uso de Cateter Venoso Central de Inserção Periférica (PICC) em recém-nascidos. Enfermería global, 24(4), 10-18.

Montes, S. F., Teixeira, J. B. A., Barbosa, M. H., \& Barichello, E. (2011). Ocorrência de complicações relacionadas ao uso de Cateter Venoso Central de Inserção Periférica (PICC) em recém-nascidos. Enfermería global, 24(4), 10-18. 
Research, Society and Development, v. 10, n. 8, e11610817059, 2021

(CC BY 4.0) | ISSN 2525-3409 | DOI: http://dx.doi.org/10.33448/rsd-v10i8.17059

Nobre, K. S. S., Cardoso, M. V. L. M. L., Teixeira, J. L., Lopes, M. M. C. O., \& Fontenele, F. C. (2016). Cateter central de inserção periférica e dissecção venosa em bebês: estudo descritivo. Online Brazilian Journal of Nursing, 15(2), 215-225.

Pereira Ferreira, C., Lemos Querido, D., Moreira Christoffel, M., Saraiva de Almeida, V., Andrade, M., \& Camilo Leite, H. (2020). The use of peripherally inserted central venous catheter in the Neonatal Intensive Care Unit. Revista Eletronica de Enfermagem, 22.

Pereira, H. P., Makuch, D. M. V., Freitas, J. S., Secco, I. L., \& Danski, M. T. R. (2020). Cateter central de inserção periférica: práticas de enfermeiros na atenção intensiva neonatal. Enfermagem em Foco, 11(4).

Rangel, R. J. M., Castro, D. S. D., Amorim, M. H. C., Zandonade, E., Christoffel, M. M., \& Primo, C. C. (2019). Práticas de inserção, manutenção e remoção do cateter central de inserção periférica em neonatos. Rev. pesqui. cuid. fundam. (Online), 278-284.

Silva, K. L. D. (2018). A equipe de enfermagem no manuseio do cateter central inserção periférica PICC. In A equipe de enfermagem no manuseio do cateter central inserção periférica PICC (pp. 43-43).

Souza, R. R. B. D., Alves, V. H., Rodrigues, D. P., Dames, L. J. P., Medeiros, F. D. V. A., \& Paiva, E. D. (2016). O conhecimento do enfermeiro sobre cateter central de inserção periférica: estudo descritivo. Online braz. j. nurs. (Online), 21-31. 